\title{
Coordination of the Sub-Regions of the Supraspinatus and Deltoid Muscles During Shoulder Scaption: a Shear Wave Elastography Study
}

\author{
K. Hoshikawa' ${ }^{1}$ T. Yuri ${ }^{1}{ }^{2}$, N. Mura ${ }^{1}$, H. Giambini ${ }^{2}$, Y. Kiyoshige $^{1}$ \\ 1 Graduate School of Health Sciences, Yamagata Prefectural University of Health Sciences, Yamagata, Japan \\ 2 Department of Biomedical Engineering, The University of Texas at San Antonio, San Antonio, Texas, U.S.A.
}

\section{CORRESPONDING AUTHOR:}

Kyosuke Hoshikawa

Graduate School of Health Sciences

Yamagata Prefectural

University of Health Sciences

260 Kamiyanagi, Yamagata, Japan,

990-2212

E-mail: d.kyosuke.hoshikawa@yachts.ac.jp

DOI:

10.32098/mltj.03.2021.22

LEVEL OF EVIDENCE: 2B

\begin{abstract}
SUMMARY
Background. Understanding the functional role of the supraspinatus (SSP) and deltoid muscles during shoulder motion is a basis for understanding rotator cuff pathology and for the development of appropriate rehabilitation protocols. The purpose of this study was to elucidate the coordination, focusing on activation timing, of the sub-regions of the SSP muscle and of the middle region of deltoid muscle during scapular plane abduction (scaption) using shear wave elastography (SWE).

Methods. Twelve healthy male volunteers without any restrictions in their shoulder joints, altered posture or scapulothoracic dyskinesis were recruited to this study. Participants were instructed to sit on a chair with their back against the back-rest. Measurements of the non-dominant arm were obtained at rest and during isometric contraction at a neutral position and every $15^{\circ}$ interval from $30^{\circ}$ to $150^{\circ}$ during scaption. Muscle activity was defined as the difference in SWE-measured stiffness between resting outcomes and those measured during muscle contraction $(\Delta \mathrm{E}=$ stiffness during contraction - stiffness at rest).

Results. The anterior-middle sub-region of the SSP muscle and the middle region of the deltoid muscle presented a mountain-shape type curve with peaks at $60^{\circ}(146.2 \pm$ $26.6 \mathrm{kPa})$ and at $90^{\circ}(142.0 \pm 25.9 \mathrm{kPa})$, respectively. The anterior-superficial sub-region of SSP muscle peaked at $30^{\circ}(102.1 \pm 27.4 \mathrm{kPa})$, linearly decreasing thereafter. Conclusions. The anterior-superficial sub-region of the SSP muscle showed activity during initial range of motion, while the anterior-middle sub-region showed activity at early mid-range. On the contrary, the middle region of the deltoid muscle showed increased SWE-measured activity at late mid-range. A more refined approach focusing on the muscles sub-regions may lead to improved rehabilitation protocols.
\end{abstract}

\section{KEY WORDS}

Coordination; deltoid; shear-wave elastography; sub-region; supraspinatus.

\section{BACKGROUND}

The rotator cuff muscles are crucial in limiting translation of the humeral head on the glenoid fossa, induced by the contraction of the deltoid muscle, and provide glenohumeral joint congruency by joint compression during shoulder motion (1-3). The middle region of deltoid muscle is the prime mover and migrates the humeral head superiorly on the glenoid fossa, while the SSP muscle predominantly applies joint compression during scapular plane abduction (scaption) $(1,3)$. Kinematic and electromyography $(E M G)$ studies have investigated the coordination of the deltoid and SSP muscles during shoulder scaption motion $(4,5)$, and showed that the activation timing between the SSP muscle and the middle region of the deltoid muscle differed, and normal motion was not achieved with a pathological SSP muscle (6).

Recent cadaveric studies have demonstrated that the SSP muscle has a complex morphology (7-10). Roh et al. divided the SSP muscle into anterior and posterior regions, based on their attachment to different sections of the internal tendon, and suggested that the anterior region is responsible for force 
production while the posterior region for an adjustment of tension (7). Kim et al. subdivided the anterior and the posterior regions into superficial, middle and deep sub-regions each based on fiber bundle arrangement (8). EMG studies have evaluated the functions of the morphologically divided regions $(11,12)$. EMG data were obtained from the anterior and posterior regions suggesting that the anterior region was a mover and the posterior region acted as stress control for the anterior region $(11,12)$. Understanding the functional roles of these sub-regions in relation to deltoid muscle activity will complement our understanding of shoulder motion and function.

Shear wave elastography (SWE) is an ultrasound technique that has been implemented to obtain quantitative estimations of tissue shear modulus, as a surrogate for tissue stiffness. Sasa- ki et al. reported a linear relationship between muscle shear modulus and tetanic muscular force produced by electrical stimulation in vivo (13). During passive muscle stretching, Koo et al. showed that the SWE elasticity increased when the muscle was being stretched and that the SWE elasticity was linearly correlated with passive resistance force (14). Hoshikawa et al., using SWE, demonstrated the different behavior of three partitions of infraspinatus muscle during scaption in vivo (15). Hatta et al. demonstrated that the four subregions of SSP muscle and five subregions of deltoid muscle presented distinct characteristics during passive motion using SWE in vitro $(16,17)$. The functions of the sub-regions of the SSP and infraspinatus muscles in previous studies using ultrasound elastography are summarized in table I. These studies

Table I. Functions of the sub-regions of the infraspinatus and supraspinatus muscles in previous studies using ultrasound elastography (a) Infraspinatus muscle. (b) Supraspinatus muscle.

(a)

\begin{tabular}{|c|c|c|c|c|c|}
\hline \multirow{3}{*}{$\begin{array}{l}\text { Author } \\
\text { (year) }\end{array}$} & \multirow[t]{3}{*}{ Measurements } & \multirow[t]{3}{*}{ Tasks } & \multicolumn{3}{|c|}{ Function } \\
\hline & & & \multicolumn{3}{|l|}{ sub-regions } \\
\hline & & & superior & middle & inferior \\
\hline $\begin{array}{l}\text { Kuwahara et al. } \\
(2017)\end{array}$ & $\begin{array}{l}\text { Strain } \\
\text { elastography }\end{array}$ & $\begin{array}{l}\text { External rotation in } \\
70^{\circ} \text { abduction } \\
\text { and } 30^{\circ} \text { of horizontal } \\
\text { abduction }\end{array}$ & Abductor & External rotator & $\begin{array}{l}\text { External rotator } \\
\text { abductor }\end{array}$ \\
\hline
\end{tabular}

(b)

\begin{tabular}{|c|c|c|c|c|c|c|c|c|}
\hline \multirow{3}{*}{$\begin{array}{l}\text { Author } \\
\text { (year) }\end{array}$} & \multirow[t]{3}{*}{ Measurements } & \multirow[t]{3}{*}{ Tasks } & \multicolumn{6}{|c|}{ Function } \\
\hline & & & \multicolumn{6}{|l|}{ Sub-Regions } \\
\hline & & & \multicolumn{3}{|l|}{ Anterior } & \multicolumn{3}{|l|}{ Posterior } \\
\hline $\begin{array}{l}\text { Yuri et al. } \\
\text { (2017) }\end{array}$ & $\begin{array}{l}\text { Strain } \\
\text { elastography }\end{array}$ & $\begin{array}{l}\text { External rotation } \\
\text { in } 90^{\circ} \text { abduction }\end{array}$ & $\begin{array}{l}\text { Force } \\
\text { production } \\
\text { for abduction }\end{array}$ & $\begin{array}{l}\text { Force } \\
\text { production } \\
\text { for abduction }\end{array}$ & $\begin{array}{l}\text { Force } \\
\text { production } \\
\text { for } \\
\text { abduction }\end{array}$ & $\begin{array}{l}\text { Maintain } \\
\text { tension }\end{array}$ & $\begin{array}{l}\text { Maintain } \\
\text { tension }\end{array}$ & $\begin{array}{l}\text { Force } \\
\text { production } \\
\text { for abduction } \\
\text { and external } \\
\text { rotation }\end{array}$ \\
\hline
\end{tabular}

\begin{tabular}{llll}
\hline $\begin{array}{l}\text { Kuwahara } \\
\text { et al. }\end{array}$ & Strain & External & Abductor \\
(2017) & elastography & rotation in & \\
& & $70^{\circ}$ abduction & \\
& & and $30^{\circ}$ of & \\
& horizontal & \\
& & abduction & \\
& &
\end{tabular}

External rotator 
suggest the feasibility of SWE to elucidate the distinct behaviors of the subregions of the SSP and deltoid muscles during scaption. Different muscle activation timing during scaption motion would lead to sub-region specific therapeutic strategies in degenerated or pathological muscles. The purpose of this study was to elucidate the coordination, focusing on activation timing, of the sub-regions of the SSP muscle and the middle region of the deltoid muscle during scaption using SWE. Outcomes from this process would provide an in-depth understanding of the contribution of the sub-regions and muscles to the shoulder scaption motion.

\section{MATERIALS AND METHODS}

\section{Participants}

A power analysis was performed a-priori to calculate the sample size needed for one-way analysis of variance $($ ANOVA) with repeated measures [effect size $=0.25, \alpha$ error $=0.05$, power $=0.8]$ using $G^{*}$ power 3.1 software (Heinrich Heine University, Duesseldorf, Germany) (18). Thus, twelve healthy male volunteers without any restrictions in their shoulder joints, altered posture or scapulothoracic dyskinesis were recruited to this study after approval by our Institutional Ethics Review Board, which was met the ethical standards of the journal (19). Their mean age, body weight, and height were $20 \pm 1$ years old, $60 \pm 3 \mathrm{~kg}$ and $170 \pm 4 \mathrm{~cm}$, respectively. Written informed consent was obtained from all participants.

\section{Experimental protocol}

Participants were instructed to sitting on a chair with their back against the back-rest and hip and knee angle at $90^{\circ}$ without pelvic posterior tilt. Investigator then checked the participants posture with their arm by side whether there are scapula dyskinesis, thoracic kyphosis and forward head (20). Before the measurement, participants were instructed warm-up exercises and stretching as well as scapular stabilizing muscles for muscular imbalance correction (21). Shoulder (SSP and middle region of the deltoid muscle) measurements of the non-dominant arm were obtained at rest and during isometric contraction at a neutral position and every $15^{\circ}$ interval from $30^{\circ}$ to $150^{\circ}$ during scapular plane abduction (scaption). A custom-built fixture was designed to allow for resting of the arm at each shoulder interval, while also allowing for active measurements. Resting measurements were obtained by having the participants place their arm at specific intervals on the fixture; active isometric contraction measurements were obtained by moving the fixture back from the resting position and instructing the participants to hold their arm against gravity in the air (figure 1). Arm postures, muscular conditions (at rest, during contraction), and sub-regions of muscle were randomized. An Aixplorer ultrasound system (Supersonic Imagine, Aix-en-Provence, France) and a 15-4 MHz linear array probe (SL 15-4) were used to obtain shear modulus $(\mathrm{kPa})$, as a surrogate for stiffness. The anterior-superficial and anterior-middle sub-regions of the SSP muscle, as determined by Kim (8), and the middle region of the deltoid muscle were identified based on their distinct muscle fiber orientations using B-mode imaging (figure 2). One investigator (K.H.) obtained a continuous SWE imaging video for each sub-region of muscle, then three random images were chosen from the video for analysis and circular regions of interest (ROI) of $4 \mathrm{~mm}$ in diameter were positioned center of muscle fiber each sub-region of muscle to obtain stiffness outcomes while looking at the B-mode maps. This process was repeated for each arm position. Muscle activity values were defined as the difference in stiffness outcomes between the active muscle contractions and the stiffness values at rest $(\Delta \mathrm{E}=$ stiffness during contraction - stiffness at rest) $(15,22)$. Muscle activity values were obtained for each shoulder position.

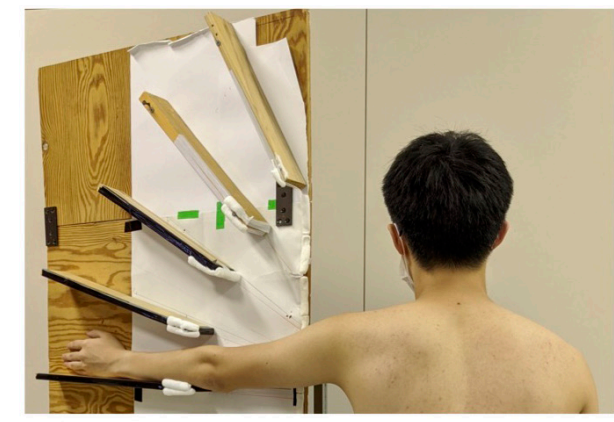

at rest

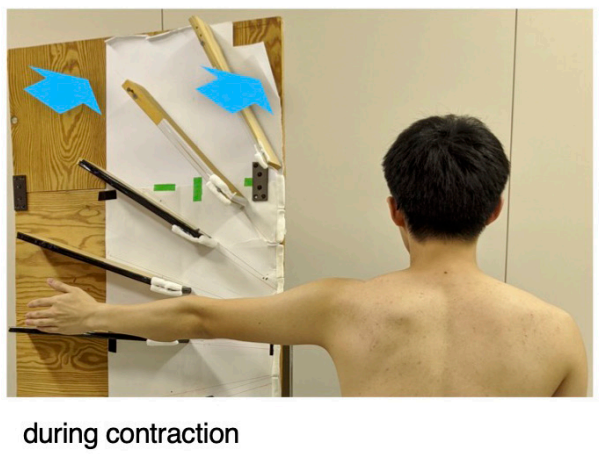

Figure 1. A resting position was attained by having the participants place their arm at specific intervals on the fixture; active isometric contraction measurements were obtained by moving the fixture buck from the resting position and instructing them to hold their arm against gravity in the air, at every $15^{\circ}$ intervals from $30^{\circ}$ to $150^{\circ}$ during abduction in scapular plane (scaption). 


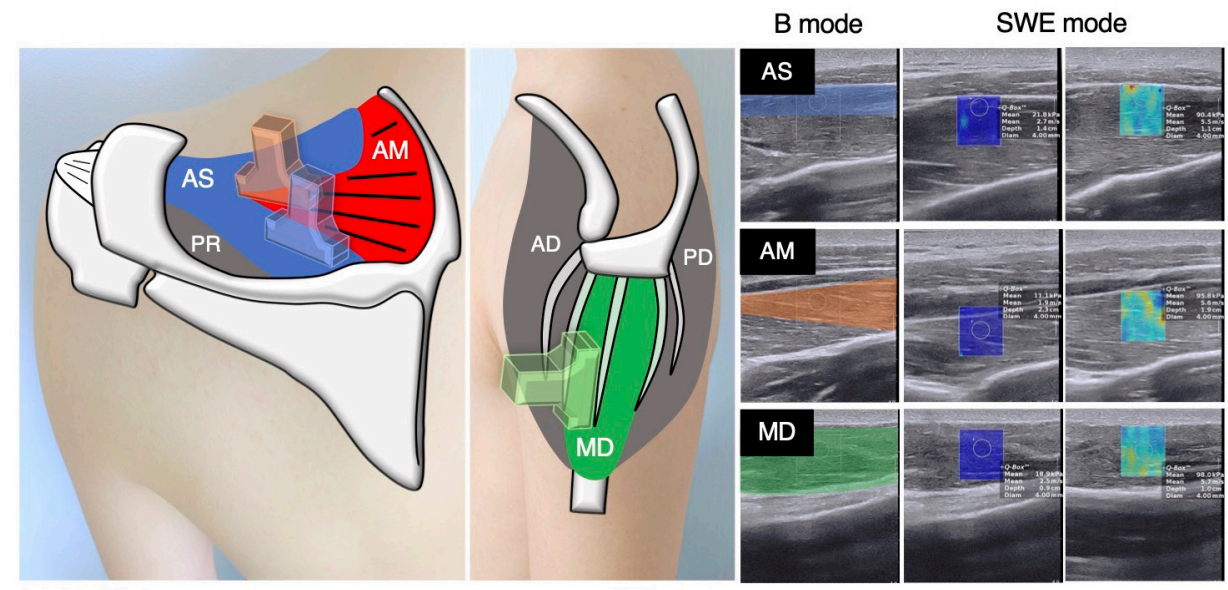

Figure 2. Probe orientations for the anterior-superficial (AS) sub-region, anterior-middle (AM) sub-region of the supraspinatus muscle and middle region of the deltoid muscle (MD). PR; posterior region of the supraspinatus muscle, AD; anterior region of the deltoid muscle, PD; posterior region of the deltoid muscle.

\section{Statistical analysis}

SPSS statistical software (version 24.0; SPSS, Chicago, IL, USA) was used for all the statistical analyses. Mean (SD) stiffness values across the twelve participants for each sub-region and shoulder position were computed. Intra-observer reliability of three images between twelve participants for each muscular sub-region and scaption angle was examined using intraclass correlation coefficient $\left(\mathrm{ICC}_{1,3}\right)$. Reliability was classified as poor (less than 0.50 ), moderate (between 0.50 and 0.75 ), good (between 0.75 and 0.90 ), and excellent (greater than 0.90) (23). One-way ANOVA with repeated measures were used to evaluate differences in measurement outcomes among nine angles of scaption in each sub-region. Paired Student's t-test with Bonferroni revision was used as post-hoc analysis to identify levels of difference among scaption angles when warranted. Statistical significance was set as $p<0.05$.

\section{RESULTS}

ICC $_{1,3}$ values among overall measurements were $>0.910$, indicating excellent reliability. Figure 3 shows the quantitative stiffness outcomes (rest, contraction, and activity value) for each muscle sub-region at various scaption angles. Example SWE images during contraction are also shown for reference. Activity values for the anterior-middle $(p<0.001$, $\mathrm{F}=57.062)$ and anterior-superficial $(p<0.001, \mathrm{~F}=41.301)$ sub-regions of the SSP muscle and the middle region of the deltoid muscle $(p<0.001, \mathrm{~F}=29.449)$ were influenced variously by scaption angle. Mean (SD) stiffness values (rest, contraction, and activity value) across the twelve participants for each sub-region and shoulder position and statistical outcomes for post-hoc test are shown in table II.
Activity outcomes of the anterior-middle sub-region of the SSP muscle (table II a) increased from $30^{\circ}$ up-to $60^{\circ}$ of scaption, reaching a value of $146.2 \pm 26.6 \mathrm{kPa}$. Activity of this muscle sub-region linearly decreased after $60^{\circ}$ with increasing elevation angles, resulting in a stiffness value of $43.2 \pm$ $21.3 \mathrm{kPa}$ at $150^{\circ}$. The peak stiffness value at $60^{\circ}$ was significantly higher than the values measured at all other angles $(p<0.002)$, except at $45^{\circ}(p=0.244)$. At angles $>75^{\circ}$, the activity values of this sub-region continued to decrease with significant differences between the activity values at adjacent arm position $(p<0.004)$, except between $105^{\circ}$ and $120^{\circ}(p=$ $0.059)$. Values obtained at $105^{\circ}$ and higher scaption angles were significantly lower than that at $30^{\circ}(p<0.019)$.

On the other hand, muscle activity outcomes of the anterior-superficial sub-region of the SSP muscle (table II b) were highest at $30^{\circ}(102.1 \pm 27.4 \mathrm{kPa})$ and linearly decreased with increasing shoulder angle position. The peak stiffness value at $30^{\circ}$ was significantly higher than the values measured at all other angles $(p<0.029)$. There were no statistical differences between the activity values at adjacent arm position at angles $>90^{\circ}(p>0.394)$.

Finally, activity values of the middle region of the deltoid muscle (table II c) showed a prominent mountain-shape with peaks of $142.0 \pm 25.9 \mathrm{kPa}$ at $90^{\circ}$. The peak value was significantly higher than the values at all other angles $(p<$ 0.007 ). The activity values of this region decreased with significant differences between values at adjacent arm position from $90^{\circ}$ to $135^{\circ}(p<0.038)$, while there was no significant difference between $135^{\circ}$ and $150^{\circ}$ measured outcomes $(p=0.155)$. Outcomes at $135^{\circ}$ and $150^{\circ}$ were higher than the lowest value of $63.1 \pm 22.7 \mathrm{kPa}$ observed at $30^{\circ}$, but not significant. 


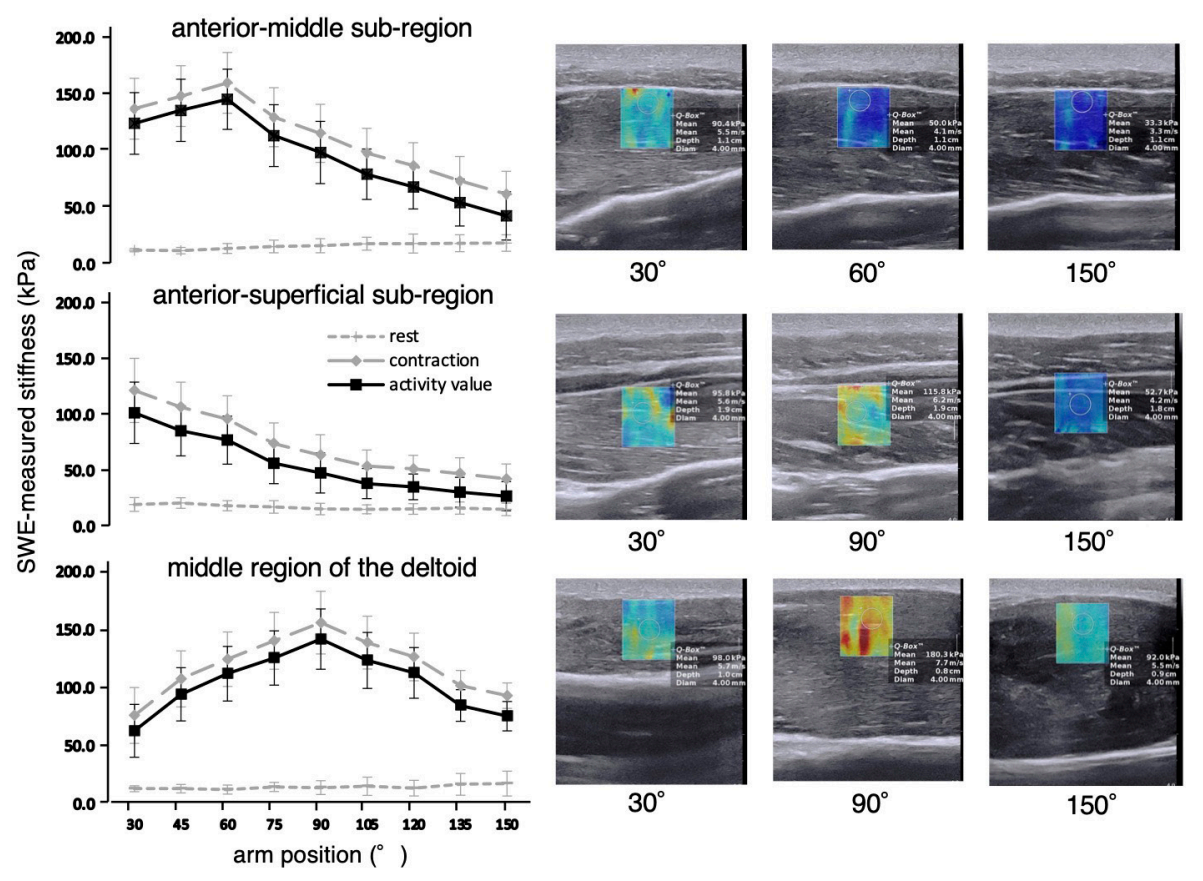

Figure 3. SWE-measured stiffness outcomes (rest, contraction, activity) and example images from the single participant for each muscle sub-region during contraction at various scaption angles.

Table II. Mean (SD) values for SWE-measured stiffness at the various arm positions and conditions (rest, contraction and activity value). (a) Anterior-middle sub-region of the supraspinatus muscle. (b) Anterior-superficial sub-region of the supraspinatus muscle. (c) Middle region of the deltoid muscle.

(a)

\begin{tabular}{|c|c|c|c|c|c|c|c|c|c|c|}
\hline & $30^{\circ}$ & $45^{\circ}$ & $60^{\circ}$ & $75^{\circ}$ & $90^{\circ}$ & $105^{\circ}$ & $120^{\circ}$ & $135^{\circ}$ & $150^{\circ}$ & $p$-value \\
\hline $\begin{array}{l}\text { Activity } \\
\text { value }\end{array}$ & $\begin{array}{l}124.8 \\
(27.2)\end{array}$ & $\begin{array}{l}136.4 \\
(27.4)\end{array}$ & $\begin{array}{l}146.2 \\
(26.6)\end{array}$ & $\begin{array}{l}114.1 * \\
(27.3)\end{array}$ & $\begin{array}{l}99.2^{*} \\
(27.5)\end{array}$ & $\begin{array}{l}80.0 * \# \\
(22.3)\end{array}$ & $\begin{array}{l}68.8 \# \\
(19.5)\end{array}$ & $\begin{array}{l}55.1^{* \#} \\
(20.8)\end{array}$ & $\begin{array}{l}43.2 * \# \\
(21.3)\end{array}$ & \multirow{2}{*}{$\begin{array}{l}*: \text { significantly lower than the value at } \\
\text { adjacent arm position } \\
(\mathrm{p}<0.004) \\
\#: \text { significantly lower than the value at } \\
30^{\circ}(\mathrm{p}<0.019)\end{array}$} \\
\hline Contraction & $\begin{array}{c}137.9 \\
(26.9)\end{array}$ & $\begin{array}{l}149.0 \\
(26.8)\end{array}$ & $\begin{array}{l}160.7 \\
(26.0)\end{array}$ & $\begin{array}{l}130.3 \\
(26.0)\end{array}$ & $\begin{array}{l}116.1 \\
(25.6)\end{array}$ & $\begin{array}{l}98.7 \\
(21.8)\end{array}$ & $\begin{array}{l}87.7 \\
(20.2)\end{array}$ & $\begin{array}{l}74.2 \\
(21.5)\end{array}$ & $\begin{array}{l}62.5 \\
(20.1)\end{array}$ & \\
\hline
\end{tabular}

(b)

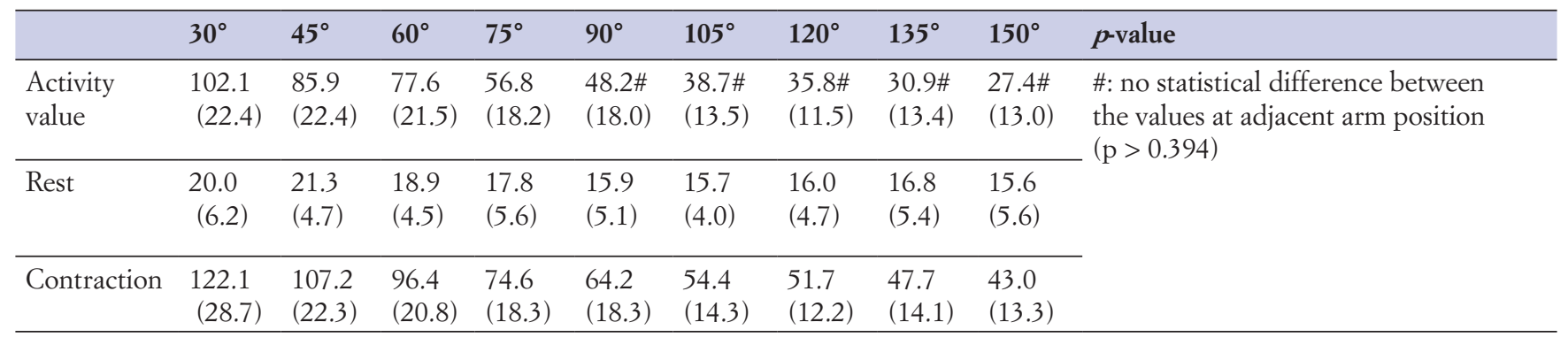




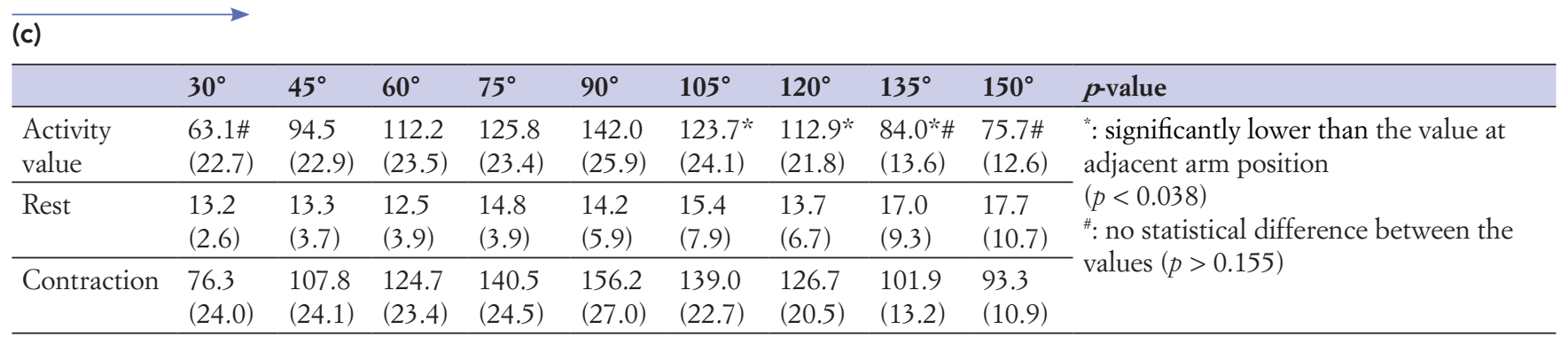

Although not significant, the stiffness at rest of each muscle sub-region changed at various scaption angles. The resting values of the anterior-middle sub-region of the SSP muscle was lowest at $45^{\circ}$ and tended to increase from $60^{\circ}$ to $90^{\circ}$, remaining almost constant from $105^{\circ}$ with increasing elevation angle. Similarly, the resting values of the middle region of the deltoid muscle was lowest at $60^{\circ}$ and then tended to increase with increasing elevation angle. On the other hand, the resting values of the anterior-superficial sub-region of the SSP muscle tended to decrease from $30^{\circ}$ to $90^{\circ}$ and constant with higher angles.

\section{DISCUSSION}

The purpose of this study was to elucidate the behaviors, focusing on activation timing, of the SSP muscle sub-regions and the middle region of the deltoid muscle during scaption motion using SWE. Results showed activity values of the anterior-middle sub-region of the SSP muscle to present a mountain-shape pattern with a peak at $60^{\circ}$ and linearly decreasing outcomes with increasing elevation angles. Similarly, the middle region of the deltoid muscle showed a mountain-shape behavior with a peak at $90^{\circ}$ but this region continued to show statistically equivalent and higher stiffness values at end-range. On the contrary, the anterior-superficial sub-region of the SSP muscle showed a peak value at $30^{\circ}$ with linearly decreasing outcomes until $90^{\circ}$ of scaption motion.

Previous EMG studies showed the SSP and deltoid muscles during scaption motion to present a mountain-shape activation pattern with peaks at $60^{\circ}$ and $90^{\circ}$, respectively (4, 5). EMG studies, since the sub-regions of the SSP muscle are so small and not be able to identify each sub-region due to not only difficulty in anatomical orientation but mutual interference, there has been only one study that revealed the functions of the anterior and posterior region of the SSP muscle using fine-wire EMG electrodes (24). Tthe placement of the electrode was performed under assistance of ultrasound B-mode at and inserted into the center of the SSP muscle, indicating that activation values were obtained solely from the anterior-middle sub-region of the SSP muscle. The results from these studies are consistent with our SWE outcomes from the anterior-middle sub-region of the SSP muscle and middle region of the deltoid muscle. The SSP and the deltoid muscles exert unique forces in the humeral head with scaption motion; activation of the SSP muscle results in a compressive force on the humeral head against the glenoid fossa, with a peak at $60^{\circ}$; while activation of the deltoid muscle, with a peak at $90^{\circ}$, will create a force on the humeral head inducing superior subluxation $(1,3)$. Therefore, the relationship, measured using SWE in the current study, between the anterior-middle sub-region of the SSP muscle and middle region of the deltoid muscle aligns well with these previous studies, indicating that these regions have a major significant role at $60^{\circ}$ and $90^{\circ}$ of scaption motion, respectively.

On the other hand, the activation timing of the anterior-superficial sub-region of the SSP muscle was different from that of anterior-middle subregion. The highest activity value in anterior-superficial sub-region was observed at $30^{\circ}$, resulting in a mean value of $<125 \mathrm{kPa}$. Activity values gradually decreased from $30^{\circ}$ to $90^{\circ}$, with minor changes thereafter and up-to $150^{\circ}$. The anterior-superficial sub-region of the SSP muscle may play a major role at $30^{\circ}$ of scaption. To our knowledge, this is the first study reporting high activity values in anterior-superficial sub-region of the SSP muscle at lower scaption angles.

Previous SWE studies demonstrated a linear relationship not only between stiffness and active contractile force, but a linear relationship between stiffness and passive stretching force $(13,14)$. As muscle is stretched, it will tend to increase its passive resistance to deformation. Thus, the stiffness at rest can indicate the length of muscle. Stiffness at rest from the anterior superficial sub-region was highest at $30^{\circ}$ and then gradually decreased up-to $90^{\circ}$ in this study. This indicates that the anterior superficial sub-region was gradually shortened with increasing elevation angles and approaching the resting length. The resting values of the anterior-middle sub-region of the SSP muscle and middle region of the deltoid muscle were lowest at $45^{\circ}$ and $60^{\circ}$, respectively, and increased thereafter with increasing elevating angle, i.e.: with a decrease in 
muscle length. These outcomes suggest that the muscular length positioned at $45^{\circ}$ corresponded to the resting length for the anterior-middle sub-region of the SSP muscle and the muscular length at $60^{\circ}$ corresponded to the resting length for the middle region of the deltoid muscle. It is important to note that the force generated by skeletal muscles will be the summation of active and passive components. Therefore, the higher activity outcomes of the anterior-superficial sub-region of the SSP muscle from $30^{\circ}$ to $90^{\circ}$ could mainly not only be a result of active muscle contraction but a result of the tension produced by passive stretching, and values from $90^{\circ}$ to $150^{\circ}$ could indicate a major role of active muscle contraction. Activity values of the anterior-middle sub-region of the SSP muscle and middle region of the deltoid muscle were a result of mainly active muscle contraction. To utilize the passive stretching force, even it is small, is reasonable for small volume muscle, such as the anterior-superficial sub-region of the SSP.

Several studies have demonstrated that, in the setting of a rotator cuff tear, the superficial subregion of the SSP primarily atrophies, while the deep subregions mainly show fat infiltration $(25,26)$. Kim et al. showed that the fiber type distribution of the anterior superficial subregion was mainly comprised of Type II fibers, while that of the anterior middle was Type I (27). Those findings also support the different function observed between the superficial and middle subregions of the SSP muscle. Current rehabilitation protocols for shoulder related injuries were developed based on the coordinated function between the deltoid muscle and the anterior middle subregion of the SSP muscle; e.g. isometric exercise is conducted at $60^{\circ}$ abducted position. In case with the superficial subregion atrophy, for example, the exercise should be conducted at $30^{\circ}$ abducted position. The findings of the current study provide evidence to investigate the coordinated function between deltoid and anatomical subregions of the SSP muscle in pathological populations so that better rehabilitation protocols can be developed.

The presence of alterations in scapular position and in its movement patterns is could be related to various conditions altering the relationship between the glenohumeral and acromioclavicular joints, or which act on correct muscle activation and coordination. Since the scapular position on the dominant side is varied as Frizziero et al. pointed out in the various instrumental musicians (28), non-dominant side was selected for measurement in this study. We also checked the participants sitting posture with their arm by side whether there are scapula dyskinesis, thoracic kyphosis and forward head to prevent altering the muscle activation and coordination affected by posture (20). Before the measurement, participants were instructed the warmup exercises and stretching as well as scapular stabilizing muscles for muscular imbalance correction (21).
There are several limitations in this study. First, we measured only two sub-regions of the SSP muscle as determined by Kim et al., who subdivided the anterior and the posterior regions into superficial, middle and deep sub-regions each (8). The selection on these regions was based on the anterior sub-region being responsible for force production and the posterior sub-region for an adjustment of tension on the tendon (8). Similarly, Roh et al. reported the anterior region to be responsible for contractile forces, while concluding that the posterior region could not generate large contractile loads (7). A study by Hatta et al. demonstrated that deep region of the anterior compartment of the SSP muscle, beneath the internal tendon, presented different stiffness outcomes compared to the superior region (16). Although the anterior region is responsible for the contractile properties of the muscle, there are still intrinsic differences between the superficial and deep sub-regions $(10,22,26,27)$. Since the purpose of this study was to elucidate the behaviors of the SSP sub-regions and the middle region of the deltoid muscle in relation to the contractile properties of the muscle during scaption motion, we only measured the anterior-superficial and anterior-middle sub-regions of the SSP muscle. Second, holding the arm against gravity was defined as isometric contraction corresponded to the manual muscle test grade 3 in this study. While this process could be considered as a weak static muscle contraction, it allowed for the evaluation of the individual SSP sub-regions during active muscle conditions. When the tasks of daily life is assumed, specific load should be applied using such as weighing scale method (29). Therapeutic strategies focusing on the specific activation timing of the sub-regions of the pathological SSP muscles will lead to better rehabilitation outcomes.

\section{CONCLUSIONS}

The anterior-superficial sub-region of the SSP muscle showed activity during initial range of motion, while the anterior-middle sub-region showed activity at early mid-range. On the contrary, the middle region of the deltoid muscle showed increased SWE-measured activity at late mid-range. A more refined approach focusing on the muscles sub-regions may lead to improved rehabilitation protocols.

\section{ACKNOWLEDGMENTS}

We thank Dr. Tatsuya Nakanowatari for special advice in statistics. We have no conflict of interest to declare.

\section{CONFLICT OF INTERESTS}

The authors declare that they have no conflict of interests. 


\section{REFERENCES}

1. Poppen NK, Walker PS. Forces at the glenohumeral joint in abduction. Clin Orthop Relat Res 1978;135:165-70.

2. Inman VT, Saunders JB, Abbott LC Observations of the function of the shoulder joint. 1944. Clin Orthop Relat Res 1996;330:3-12.

3. Yanagawa T, Goodwin CJ, Shelburne KB, Giphart JE, Torry MR, Pandy MG. Contributions of the individual muscles of the shoulder to glenohumeral joint stability during abduction. J Biomech Eng 2008;130(2):021024.

4. Reed D, Cathers I, Halaki M, Ginn KA. Does changing the plane of abduction influence shoulder muscle recruitment patterns in healthy individuals? Man Ther 2016;21:63-8.

5. Hawkes DH, Khaiyat OA, Howard AJ, Kemp GJ, Frostick SP. Patterns of muscle coordination during dynamic glenohumeral joint elevation: An EMG study. PLoS One 2019;14(2):e0211800.

6. McMahon PJ, Jobe FW, Pink MM, Brault JR, Perry J. Comparative electromyographic analysis of shoulder muscles during planar motions: anterior glenohumeral instability versus normal. J Shoulder Elbow Surg 1996;5(2 Pt 1):118-23.

7. Roh MS, Wang VM, April EW, Pollock RG, Bigliani LU, Flatow EL. Anterior and posterior musculotendinous anatomy of the supraspinatus. J Shoulder Elbow Surg 2000;9(5):436-40.

8. Kim SY, Boynton EL, Ravichandiran K, Fung LY, Bleakney R, Agur AM. Three-dimensional study of the musculotendinous architecture of supraspinatus and its functional correlations. Clin Anat 2007;20(6):648-65.

9. Hermenegildo JA, Roberts SL, Kim SY. Innervation pattern of the suprascapular nerve within supraspinatus: a three-dimensional computer modeling study. Clin Anat 2014;27(4):622-30.

10. Yuri T, Kobayashi H, Takano Y, Yoshida S, et al. Capsular attachment of the subregions of rotator cuff muscles. Surg Radiol Anat 2019;41(11):1351-9. 11.

11. Alenabi T, Whittaker RL, Kim SY, Dickerson CR. Arm posture influences on regional supraspinatus and infraspinatus activation in isometric arm elevation efforts. J Electromyogr Kinesiol 2019;44:108-16.

12. Lulic-Kuryllo T, Alenabi T, McDonald AC, Kim SY, Dickerson CR. Sub-regional activation of supraspinatus and infraspinatus muscles during activities of daily living is task dependent. J Electromyogr Kinesiol 2020;54:102450.

13. Sasaki K, Toyama S, Ishii N. Length-force characteristics of in vivo human muscle reflected by supersonic shear imaging. J Appl Physiol (1985) 2014;117(2):153-62. 14. Koo TK, Guo JY, Cohen JH, Parker KJ. Relationship between shear elastic modulus and passive muscle force: an ex-vivo study. J Biomech 2013;46(12):2053-9.

14. Hoshikawa K, Yuri T, Giambini H, Kiyoshige Y. Shoulder scaption is dependent on the behavior of the different partitions of the infraspinatus muscle. Surg Radiol Anat 2021. Online ahead of print.
15. Hatta T, Giambini H, Itoigawa Y, et al. Quantifying extensibility of rotator cuff muscle with tendon rupture using shear wave elastography: A cadaveric study. J Biomech 2017;61:131-136.

16. Hatta T, Giambini H, Sukegawa K, et al. Quantified Mechanical Properties of the Deltoid Muscle Using the Shear Wave Elastography: Potential Implications for Reverse Shoulder Arthroplasty. PLoS One 2016;11(5):e0155102.

17. Faul F, Erdfelder E, Lang AG, Buchner A. G*Power 3: a flexible statistical power analysis program for the social, behavioral, and biomedical sciences. Behav Res Methods 2007;39(2):175-91.

18. Padulo J, Oliva F, Frizziero A, Maffulli N. Basic principles and recommendations in clinical and field Science Research: 2018 update. Muscles Ligaments Tendons J 2018;8(3):305-7.

19. McKenna L, Cornwall X, Williams S. Differences in Scapular Orientation Between Standing and Sitting Postures at Rest and in $120^{\circ}$ Scaption: A Cross-Sectional Study. PM\&R 2017;9(6):579-87.

20. Merolla G, Cerciello S, Paladini P, Porcellini G. Snapping scapula syndrome: current concepts review in conservative and surgical treatment. Muscles Ligaments Tendons J 2013;3(2):80-90.

21. Yuri T, Kuwahara Y, Fujii H, Kiyoshige Y. Functions of the subregions of the supraspinatus muscle. Clin Anat 2017;30(3):347-51.

22. Koo TK, Li MY. A Guideline of Selecting and Reporting Intraclass Correlation Coefficients for Reliability Research. J Chiropr Med 2016;15(2):155-63.

23. Kim SY, Ko JB, Dickerson CR, Collins DF. Electromyographic investigation of anterior and posterior regions of supraspinatus: a novel approach based on anatomical insights. Int Biomech 2017;4(2):60-7.

24. Kim SY, Sachdeva R, Li Z, Lee D, Rosser BW. Change in the Pathologic Supraspinatus: A Three-Dimensional Model of Fiber Bundle Architecture within Anterior and Posterior Regions. Biomed Res Int 2015;564825.

25. Trevino Iii JH, Yuri T, Hatta T, Kiyoshige Y, Jacobs PM, Giambini $H$. Three-dimensional quantitative measurements of atrophy and fat infiltration in sub-regions of the supraspinatus muscle show heterogeneous distributions: a cadaveric study. Arch Orthop Trauma Surg 2021. Online ahead of print.

26. Kim SY, Lunn DD, Dyck RJ, Kirkpatrick LJ, Rosser BW. Fiber type composition of the architecturally distinct regions of human supraspinatus muscle: a cadaveric study. Histol Histopathol 2013;28(8):1021-1028.

27. Frizziero A, Gasparre G, Corvo S, et al. Posture and scapular dyskinesis in young bowed string instrumental musicians ali Antonio Frizziero. Muscles Ligaments Tendons J 2018;8(4):507-12.

28. Collin P, Banarji BH, Denard PJ, Kherad O, Lädermann A. Comparison of shoulder strength assessment in scaption with an isometric dynamometer and a weighing machine: a pilot study. JSES Open Access. 2018;2(2):141-3. 